\title{
Environmental tests of the flight GLAST LAT tracker towers
}

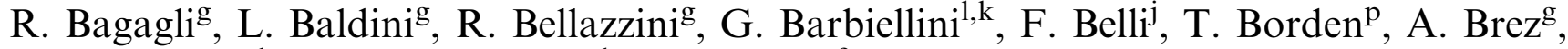 \\ M. Brigida ${ }^{\mathrm{a}, \mathrm{b}}$, G.A. Caliandro ${ }^{\mathrm{a}, \mathrm{b}}$, C. Cecchi $^{\mathrm{e}, \mathrm{f}}$, J. Cohen-Tanugi ${ }^{\mathrm{p}}$, A. De Angelis ${ }^{\mathrm{m}, \mathrm{n}}$,

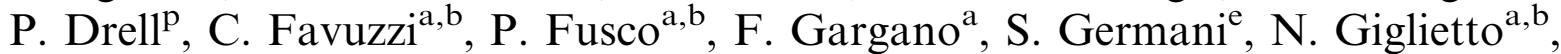

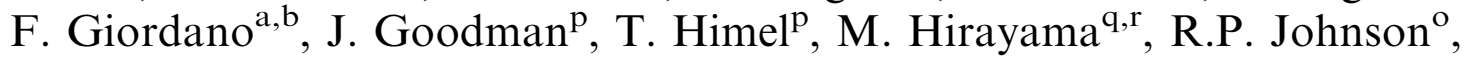 \\ H. Katagiri ${ }^{\mathrm{s}}$, J. Kataoka ${ }^{\mathrm{t}}$, N. Kawai ${ }^{\mathrm{t}}$,W. Kroeger ${ }^{\mathrm{p}}$, J. Ku ${ }^{\mathrm{p}}, \mathrm{M}$. Kuss ${ }^{\mathrm{g}}$, \\ L. Latronico ${ }^{\mathrm{g}}$, F. Longo ${ }^{1, \mathrm{k}}$, F. Loparco ${ }^{\mathrm{a}, \mathrm{b}}$, P. Lubrano ${ }^{\mathrm{e}}$, B. Marangelli ${ }^{\mathrm{a}, \mathrm{b}}$, \\ F. Marcucci, ${ }^{\mathrm{e}, \mathrm{f}}$, M. Marchetti ${ }^{\mathrm{j}}$, M.M. Massai ${ }^{\mathrm{g}, \mathrm{h}}$, M.N. Mazziotta ${ }^{\mathrm{a}, *}$, M. Minori ${ }^{\mathrm{j}}$, \\ M. Minuti ${ }^{\mathrm{g}}$, N. Mirizzi ${ }^{\mathrm{a}, \mathrm{b}}$, M. Mongelli ${ }^{\mathrm{a}}$, C. Monte ${ }^{\mathrm{a}, \mathrm{b}}$, A. Morselli $^{\mathrm{j}}$, D. Nelson $^{\mathrm{p}}$, \\ M. Nordby ${ }^{\mathrm{p}}$, N. Omodei ${ }^{\mathrm{g}}$, M. Pepe ${ }^{\mathrm{e}}$, M. Pesce-Rollins ${ }^{\mathrm{g}}$, S. Rainò ${ }^{\mathrm{a}, \mathrm{b}, * *}$,

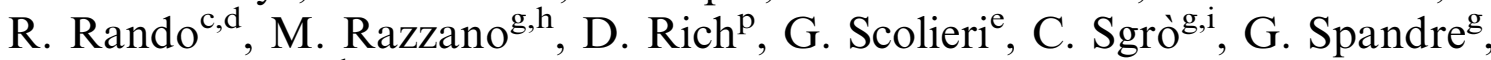 \\ P. Spinellia, ${ }^{\mathrm{a},}$, M. Sugizaki ${ }^{\mathrm{p}}$, H. Takahashi ${ }^{\mathrm{s}}$, A. Tenze ${ }^{\mathrm{g}}$, C. Young $^{\mathrm{p}}$ \\ ${ }^{a}$ Istituto Nazionale di Fisica Nucleare Sezione di Bari, Via E. Orabona 4, 70126 Bari, Italy \\ ${ }^{\mathrm{b}}$ Dipartimento Interateneo di Fisica dell'Università e Politecnico di Bari, Via G. Amendola 173, 70126 Bari, Italy \\ ${ }^{\mathrm{c}}$ Istituto Nazionale di Fisica Nucleare Sezione di Padova, Via Marzolo 8, 35131 Padova, Italy \\ ${ }^{\mathrm{d}}$ Dipartimento di Fisica dell'Università di Padova, Via Marzolo 8, 35131 Padova, Italy \\ ${ }^{\mathrm{e}}$ Istituto Nazionale di Fisica Nucleare Sezione di Perugia, Via A. Pascoli, 06123 Perugia, Italy \\ ${ }^{\mathrm{f}}$ Dipartimento di Fisica dell'Università di Perugia, Via A. Pascoli, 06123 Perugia, Italy \\ ${ }^{\mathrm{g}}$ Istituto Nazionale di Fisica Nucleare Sezione di Pisa, Largo B. Pontecorvo 3, 56127 Pisa, Italy

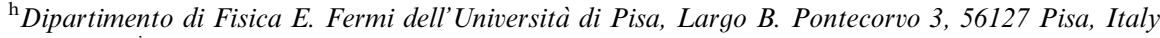 \\ ${ }^{i}$ Scuola Normale Superiore di Pisa, Piazza dei Cavalieri 7, 56126 Pisa, Italy \\ ${ }^{\mathrm{j}}$ Istituto Nazionale di Fisica Nucleare Sezione di Roma Tor Vergata, Via della Ricerca Scientifica 1, 00133 Roma, Italy \\ ${ }^{\mathrm{k}}$ Istituto Nazionale di Fisica Nucleare Sezione di Trieste, Via Valerio 2, 34012 Trieste, Italy \\ ${ }^{1}$ Dipartimento di Fisica dell'Università di Trieste, Via Valerio 2, 34012 Trieste, Italy \\ ${ }^{\mathrm{m}}$ Istituto Nazionale di Fisica Nucleare Gruppo Collegato di Udine, Via delle Scienze 208, 33100 Udine, Italy \\ ${ }^{\mathrm{n}}$ Dipartimento di Fisica dell'Università di Udine, Via delle Scienze 208, 33100 Udine, Italy \\ ${ }^{\circ}$ Santa Cruz Institute for Particle Physics, University of California, 1156 High Street, Santa Cruz, CA 95064, USA \\ ${ }^{\mathrm{p}}$ Stanford Linear Accelerator Center (SLAC), Menlo Park, CA 94025, USA \\ ${ }^{\mathrm{q}}$ CRESST and Astroparticle Physics Laboratory NASA/GSFC, Greenbelt, MD 20771, USA \\ ${ }^{\mathrm{r}}$ Department of Physics, University of Maryland, Baltimore County, 1000 Hilltop Circle, Baltimore, MD 21250, USA \\ ${ }^{\mathrm{s}}$ Hiroshima University, 1-3-1 Kagamiyama, Higashi-Hiroshima 739-8526, Japan \\ ${ }^{\mathrm{t}}$ Tokyo Institute of Technology, 2-12-1 Ohokayama Meguro, Tokyo 152-8551, Japan
}

\begin{abstract}
The Gamma-ray Large Area Space telescope (GLAST) is a gamma-ray satellite scheduled for launch in 2008. Before the assembly of the Tracker subsystem of the Large Area Telescope (LAT) science instrument of GLAST, every component (tray) and module (tower) has been subjected to extensive ground testing required to ensure successful launch and on-orbit operation. This paper describes the sequence and results of the environmental tests performed on an engineering model and all the flight hardware of the GLAST LAT

\footnotetext{
*Corresponding author. Tel.: + 390805443163 ; fax: + 390805442470 .

**Also Corresponding author. Istituto Nazionale di Fisica Nucleare Sezione di Bari, Via E. Orabona 4, 70126 Bari, Italy. Tel.: +39080 5443172; fax +390805442470 .
} 
Tracker. Environmental tests include vibration testing, thermal cycles and thermal-vacuum cycles of every tray and tower as well as the verification of their electrical performance.

\section{Introduction}

The Gamma Ray Large Area Space Telescope (GLAST) is an international space mission scheduled for launch in 2008 on a Delta-II rocket. It will explore the gamma-ray sky in an energy range between $10 \mathrm{keV}$ and $300 \mathrm{GeV}$ that was mainly unexplored by previously flown instruments, [1-3]. The GLAST mission was conceived to address important outstanding questions in high energy astrophysics, many of which were raised but not answered by results from EGRET on board of the Compton Gamma Ray Observatory (CGRO) that made the first complete survey of the sky in the $30 \mathrm{MeV}$ to $10 \mathrm{GeV}$ range [4].

GLAST consists of two scientific instruments, the Large Area Telescope (LAT) [5] and the Gamma-ray Burst Monitor (GBM) [6]. The LAT is a gamma-ray telescope covering the energy range from $20 \mathrm{MeV}$ up to $300 \mathrm{GeV}$. The GBM will have a very large field of view, covering all the sky not occulted by Earth, and will provide spectral coverage of gamma-ray bursts from the lower energy limit of the LAT down to $10 \mathrm{keV}$. It includes two sets of detectors: $12 \mathrm{NaI}$ scintillators, each $12.7 \mathrm{~cm}$ in diameter by $1.27 \mathrm{~cm}$ in thickness, and two cylindrical BGO scintillators, each $12.7 \mathrm{~cm}$ in diameter and $12.7 \mathrm{~cm}$ in height.

As for all space-based instruments, an environmental verification for GLAST as a whole and for all its subsystems (see for instance Ref. [7]) is required before launch to ensure successful launch and on-orbit operation. The primary purpose of this testing is risk mitigation, since these instruments typically do not operate in a benign environment and are not accessible for repair. The main ground environmental tests for space-bound articles include thermal, structural (vibration and acoustic), and electromagnetic interference proofs.

Environmental test campaigns have always been considered a mandatory phase in the construction and verification process of satellites and space-based instruments considering the inaccessibility of the hardware in the operation environment. However, environmental tests could also play a basic role in the development and testing of future ground-based high energy physics experiments, which are becoming so complex and are so inaccessible during operation that planning and performance of adequate environmental testing is needed to ensure their correct operation.

The LAT Tracker was designed to satisfy the GLAST science requirements, while fitting within the constraints of a Delta-II rocket payload fairing. The Tracker mechanical structure must support and protect the detectors during launch. It must also provide passive cooling paths for the waste heat of the electronics, survive worst-case temperature extremes, and serve as a shield from electromagnetic interference. The Tracker design is fully described in Ref. [8].

This article focuses on the procedures and results of the environmental tests on the LAT Tracker trays and towers started in 2003 and completed in October 2005. Vibration, thermal-cycles and thermal-vacuum tests were successfully performed on all the items. The activity on the flight hardware also included electrical tests necessary to verify the detectors performance before, during and after the environmental test sequence. This activity was preceded by a compelling study on pre-engineering and engineering models that were tested at qualification levels in order to validate the design and verify workmanship before building the flight modules. The results obtained on the engineering model units were of primary importance in the definition and the approval of the final design and they were also used to optimize the plans and procedures of the tests on the flight modules that are explained in the following sections.

\section{Environmental test strategy}

Verification tests are usually performed at different phases during the construction sequence. Lower level components or subassemblies are individually tested over the widest range as is appropriate for that level of testing. Higher assembly levels in subsystems are tested in a narrower range. At the final level, the instrument system is tested in the narrowest range, which is appropriate for system level acceptance testing. This test methodology ensures that a component or subassembly never experiences a more extreme level than what was previously tested.

Environmental tests can be classified on the basis of the hardware assembly level (starting from the component or unit level of assembly through the system level) and on the type of hardware being tested. In the latter case, two major categories of hardware can be considered:

- prototype hardware: new design hardware, subject to a design qualification test program and not intended for flight, such as engineering model or qualification hardware;

- flight hardware: hardware to be used operationally in space, including proto-flight, flight, flight-spare, re-flight. 
Table 1

General verification test levels

\begin{tabular}{lll}
\hline Test article & Test level & Purpose \\
\hline Engineering model & Development & Proof of concept \\
Prototype & Qualification & Design qualification \\
Proto-flight & Proto-qualification & Flight hardware of new design \\
Flight & Acceptance & Workmanship \\
\hline
\end{tabular}

A summary of the different verification test levels is reported in Table 1.

Qualification tests demonstrate that the design, manufacturing process and acceptance test program produce hardware that meets mission specification requirements. To the extent possible, the test article should be produced using the same drawings, materials, tooling, manufacturing processes and level of personnel competency as the production of flight articles. The test environment is more severe than the expected load limit including a safety factor. For instance, the dynamic limit levels are typically increased by $25 \%$, the temperatures are more extreme (e.g. $\pm 10^{\circ} \mathrm{C}$ ) and eventually the number of cycles is higher than that experienced in acceptance level testing.

A proto-qualification test is a hybrid between a qualification and an acceptance test conducted on a single article. For example in thermal testing, temperature extremes are the same as or lower than qualification, but the number of thermal cycles is eventually reduced. The proto-flight hardware is available for flight.

Acceptance tests act as an environmental stress screen to precipitate incipient failures resulting from latent defects in workmanship and materials. Acceptance tests are conducted to demonstrate the acceptability of a deliverable article and verify conformance to specification requirements. Acceptance tests prove flightworthiness of the tested article.

The primary goal of environmental tests on GLAST LAT Tracker towers was to demonstrate the satisfactory performance of hardware in the expected mission environments and to verify that minimum workmanship standards were met. A collective program plan for the LAT instrument test was studied, addressing the testing to be performed at the unit/subsystem and instrument level for qualification, proto-flight and acceptance testing phases.

\section{Detector description and test articles}

The LAT is a gamma-ray telescope based on the conversion of gamma-rays into electron-positron pairs and is arranged in a $4 \times 4$ array of 16 identical towers (Fig. 1). Each tower comprises a silicon micro-strip detectors Tracker (TKR) followed by a segmented CsI calorimeter (CAL), to reconstruct the gamma-ray direction and energy. A custom-designed data acquisition module (TEM, Tower Electronics Module) is located below the calorimeter. The Tracker and the calorimeter are covered

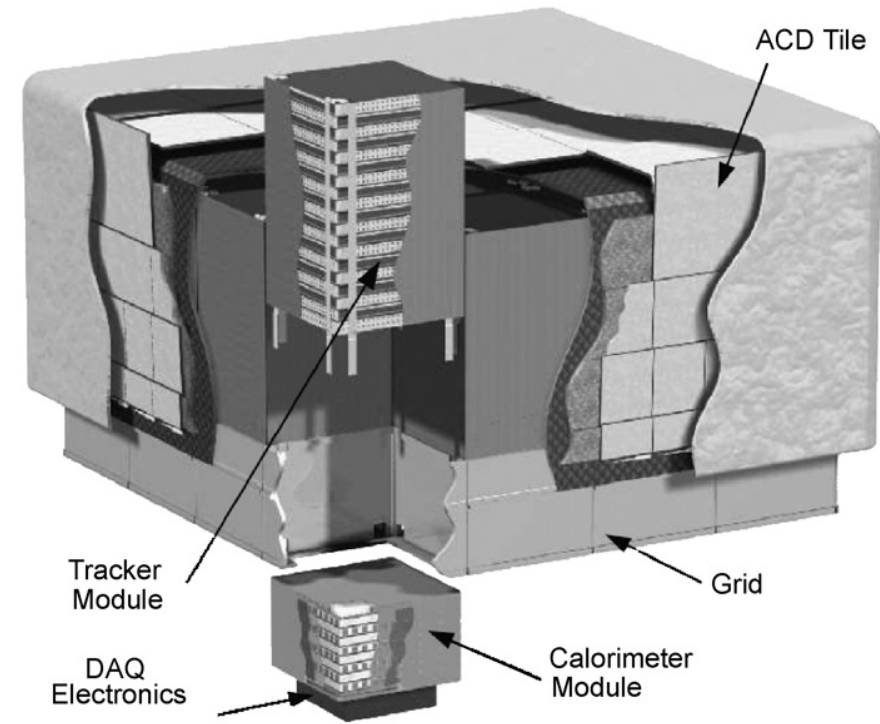

Fig. 1. Cutaway view of the LAT detector. Each tower in the $4 \times 4$ array includes a TKR and a CAL.

by a segmented scintillator anti-coincidence system (ACD), consisting of panels of plastic scintillator read out by waveshifting fibers and photo-multiplier tubes, used to reject cosmic charged particle background (electrons, protons, heavier nuclei). The ACD is also covered by a thermal blanket and micro-meteorite shield. An aluminum Grid supports the detector modules and the data acquisition system and computers, which are located below the CAL modules. The LAT detector is described in more detail in Ref. [5].

The Tracker mechanical structure has been designed to support and protect the detectors, electronics and converter foils during launch, maintaining the precise locations of the detectors while using a minimal amount of material. Each of the Tracker tower modules (TKR hereafter) is composed of a stack of 19 stiff, lightweight carbon composite panels called trays: one bottom tray, 17 mid trays, and one top tray. A tray panel is a composite structure: an aluminum honeycomb core is closed by two carbon-fiber face sheets, bonded to four carbon-carbon close-outs to form a sandwich structure for mounting payload. On the top and bottom surfaces of the tray two kapton flexible circuits are glued to supply the bias potential to the silicon microstrip detectors (SSDs). Exploded views of a TKR and a tray are shown in Figs. 2 and 3.

The silicon detectors are bonded on both sides of a panel, with the strips on top parallel to those on the bottom. One layer hosts 16 silicon detectors arranged in an array of four ladders, each ladder consisting of four wafers glued head to head and bonded together to form a single detector assembly. An array of tungsten (W) converter foils is glued to the bottom surface of all but the three lowest trays, between the panel and the flexible circuits and detectors, to match the active area of each wafer. The first 12 layers of tungsten are each $2.7 \%$ radiation length (r.1.) in thickness, while the following four layers are $18 \%$ r.l. thick 


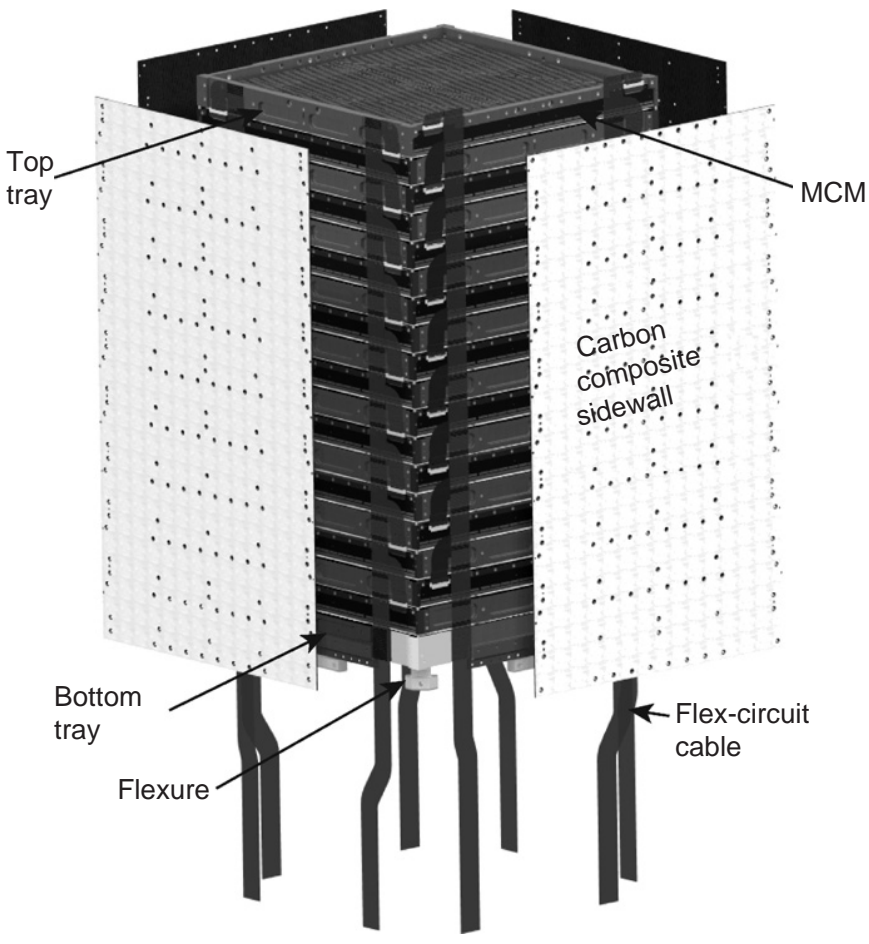

Fig. 2. Exploded view of a TKR.

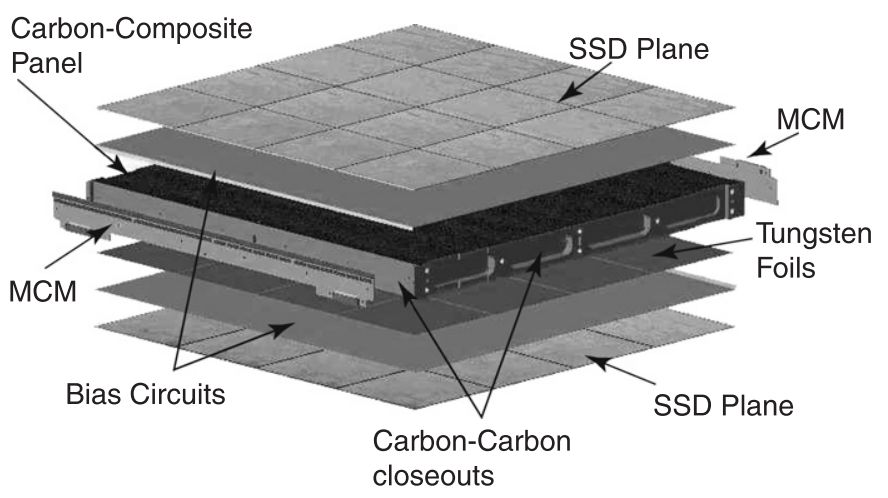

Fig. 3. Exploded view of a mid tray, illustrating the integration of detectors and electronics.

to increase the detection efficiency in the $\mathrm{GeV}$ and above energy range. Only the top and bottom trays support silicon detectors on one side only.

Every tray is rotated $90^{\circ}$ with respect to the one above or below. The detectors on the bottom of a tray combine with those on the top of the tray below to form $x, y$ pairs with a $2 \mathrm{~mm}$ gap between them and with the tungsten converter foils located just above.

The bottom tray has been designed to have a reinforced structure that is much stronger than the upper trays in order to provide the interface of the entire tower module to the Grid. As discussed in Section 6.1.3, the final design of the bottom tray followed the failure of the first vibration test of the engineering model tower, whose goal was to qualify the Tracker design.

The bottom tray close-outs are much stronger and stiffer than the others, formed by a different carbon fiber composite (M55J with a veneer of carbon-carbon bonded to it). Their corners are reinforced by titanium brackets which carry most of the load from the sidewalls into the Grid interface. Eight flexures provide the mechanical interface of the bottom tray to the Grid. There is one flexure on each corner, mounted in the corner bracket, and one at the center of each side, bolted into inserts in the tray. The flexures are oriented such that the Grid can expand and contract with temperature variations without putting significant stress into the carbon composite structure. On the other hand, the thermal interface from the bottom tray to the Grid is provided by thermal straps, composed each by two layers of copper, $0.38 \mathrm{~cm}$ thick, screwed between the sidewall and the bottom tray on the upper side and clamped between an aluminum bar and the Grid on the bottom side. More technical details can be found in Refs. [8,9].

The front-end electronics (MCM, multi chip module) are mounted on the sides of the tray panels. Eight thin flexiblecircuit cables (one on the left edge and one on the right edge of each side) connect the nine MCMs/side of a TKR to the data acquisition electronics. All power, control, data, triggers, and housekeeping signals pass through these cables. The flex cables are approximately $90 \mathrm{~cm}$ long and interface the nine MCMs read-out by each cable to the TEM. Two thermistors are mounted on the backside of each cable for monitoring the temperature profile in the TKR.

Carbon composite sidewalls are used to close the towers on the four sides, providing stiff support for the TKR with minimal scattering of particles passing from one module to another. Each sidewall consists of 12 layers of Mitsubishi K13D2U fibers with two layers of Nippon-Graphite SFY-S90A-75 fabric on each side, all bonded with CE3 cyanate-ester resin. A $25 \mu \mathrm{m}$ aluminum foil is bonded on each side to provide electrical shielding. This foil is painted black to increase the thermal emissivity of the sidewall. Moreover, the sidewalls are used to conduct the waste heat from the electronics down the tower to the Grid, through the thermal path provided by the copper straps.

The test articles used for the LAT Tracker environmental test campaign are the TKRs, and the trays from which they are built, classified as follows:

(1) an engineering model tower: completely equivalent to a flight tower from the structural point of view but using non-functional (dummy) silicon wafers. In addition the electronics modules were assembled only with resistances that simulate the power dissipation;

(2) a proto-flight tower (TKR ID A);

(3) 16 flight Tracker towers, 15 to complete the LAT assembly (TKR ID B, TKR IDs 1-7 and 9-15) and 1 flight spare (TKR ID 8 );

(4) a non-flight tower for ground testing (TKR ID 16).

The TKRs and their components (trays) are required to survive to the levels identified in the LAT Environmental 
Specification [10] according to the NASA-specified General Environmental Verification Specification (GEVS) [11] test levels. From the structural point of view, this means that the TKRs must survive to the launch environment without damage and without contact between successive SSD layers $(2 \mathrm{~mm}$ gaps) or between adjacent modules $(2.5 \mathrm{~mm}$ distance). The structure must also survive and correctly operate in vacuum over a temperature range expected during the mission.

The engineering model tower was tested to qualification levels. The proto-flight tower was also tested to levels higher than acceptance, while the other 17 TKRs were tested to acceptance levels. Among the 17 functional modules, the proto-flight and 15 of the flight articles were used to assemble the LAT Tracker, while the two spare TKRs were used to build a Calibration Unit used for a beam test at CERN in summer 2006 [12].

\section{Test plans and procedures}

The environmental test activity is based on the LAT Program Instrument Performance Verification Plan [13] that identifies all the tests and analyses required to demonstrate compliance with requirements of the GLAST LAT instrument. It addresses in a more specific way the NASA general requirements defined in GEVS and focuses on the testing to be performed at the unit/subsystem and instrument level for flight qualification, proto-flight and acceptance testing phases for the GLAST LAT case.

The environmental verification process includes mechanical tests (static and dynamic), thermal tests (thermal cycling, thermal balance, thermal vacuum), electromagnetic tests (emission and susceptibility) and electrical tests. The dynamic (vibration) tests include sine (survey, sinusoidal and quasi-static) and random vibration. The random vibration test is performed by using an acceleration spectral density (ASD) spectrum that includes the acoustic inputs plus the effects of vibration transmitted through the structure during the launch. The sine survey is a low level sinusoidal sweep to measure the fundamental frequency of the test article. The main scope of the sine survey is to help detecting eventual structural damages that may occur during the sinusoidal and random vibration tests.

The test levels are based on the LAT Environmental Specification that defines the structural, thermal, and onorbit design exposures and test environments for the LAT instrument and its subsystems. This includes launch structural loads, on-orbit thermal environments, and onorbit debris, radiation, and other environments that may affect end-of-life reliability and performance. All the Tracker tests were carried out following the plans and procedures properly defined for each test article and kind of test. The test procedures also include the fail and success criteria for each test and item. The test levels applied to the Tracker modules are summarized in Tables 2 and 3, and will be discussed in more detail in the following sections.
Table 2

Trays environmental test levels summary table

\begin{tabular}{lll}
\hline Article & \multicolumn{2}{l}{ Test at tray level } \\
\cline { 2 - 3 } & $\begin{array}{l}\text { Random vibration } \\
(2-2000 \mathrm{~Hz})\end{array}$ & Thermal cycle \\
\hline Engineering model & $14 g$ RMS & $\begin{array}{l}-30 \text { to }+50^{\circ} \mathrm{C} \\
\text { Max. } \\
\text { rate }= \pm 20^{\circ} \mathrm{C} / \mathrm{h}\end{array}$ \\
$\begin{array}{l}\text { Proto-flight tower } \\
\text { (TKR ID A) }\end{array}$ & $10 g$ RMS & $\begin{array}{l}\text { Max. }+55^{\circ} \mathrm{C} \\
\text { rate }= \pm 40^{\circ} \mathrm{C} / \mathrm{h}\end{array}$ \\
$\begin{array}{l}\text { Flight towers } \\
\text { (TKR IDs B and }\end{array}$ & Not performed & $\begin{array}{l}-15 \text { to }+45^{\circ} \mathrm{C} \\
\text { 1-15) }\end{array}$ \\
\hline
\end{tabular}

The procedures describe the test equipment, configuration, levels and a detailed test sequence, with vibration tests carried out before the thermal-vacuum tests. For the flight modules the two tests are preceded and followed by the electrical functional tests to verify the SSDs performance.

The environmental tests on the TKRs and their components have been performed in different locations (INFN labs and external companies), according to the availability of necessary instrumentation. All the tests have been developed and carried out by the LAT Tracker group in the locations chosen on the basis of the available facilities required for the testing.

Briefly, the complete environmental test sequence was carried out in the following locations:

- vibration tests on tray panels (before integration of SSDs and electronics) at Centrotecnica s.a.s. (Milan) [14];

- thermal cycles and burn-in tests carried out on MCMs at SLAC $[15,16]$;

- thermal cycles on trays, equipped with SSDs and MCMs, at room pressure in INFN laboratories;

- cosmic ray tests with stacked trays before the tower assembly, carried out in INFN laboratories;

- vibration and thermal-vacuum tests on TKRs at AleniaAlcatel (Rome) in the AIT (Assembly Integration and Test) center [17].

\section{Test sequence and performance of trays}

The environmental tests sequence on the trays has been performed at different assembly levels (see Table 2). The vibration test activity was carried out on tray panels (panel hereafter) prior to the integration of silicon detectors and electronics. Then the thermal cycles and the stacked tray tests followed the completion of the tray assembly with detectors and electronics. The environmental test activity on trays is reported in the following sections and the different tests and results are separately discussed. 
Table 3

Tracker towers environmental test levels summary table

\begin{tabular}{|c|c|c|c|}
\hline \multirow[t]{2}{*}{ Article } & \multicolumn{3}{|l|}{ Tests at tower level } \\
\hline & Sine test $(5-50 \mathrm{~Hz})$ & Random vibration $(20-2000 \mathrm{~Hz})$ & Thermal vacuum \\
\hline Engineering model & $\begin{array}{l}8.5 g(Z) \\
5.5 g(X, Y)\end{array}$ & $\begin{array}{l}6.1 g \operatorname{RMS}(Z) \\
6.6 g \operatorname{RMS}(X, Y)\end{array}$ & $\begin{array}{l}\text { Thermal balance } \\
1 \text { cycle } \\
-15 \text { to }+25^{\circ} \mathrm{C} \\
\text { Max. rate }= \pm 20^{\circ} \mathrm{C} / \mathrm{h}\end{array}$ \\
\hline $\begin{array}{l}\text { Proto-flight tower } \\
\text { (TKR ID A) }\end{array}$ & $\begin{array}{l}8.5 g(Z) \\
5.5 g(X, Y)\end{array}$ & $\begin{array}{l}6.1 g \operatorname{RMS}(Z) \\
6.6 g \operatorname{RMS}(X, Y)\end{array}$ & $\begin{array}{l}\text { Thermal balance } \\
4 \text { cycles } \\
-20 \text { to }+50{ }^{\circ} \mathrm{C} \\
\text { Max. rate }= \pm 20^{\circ} \mathrm{C} / \mathrm{h}\end{array}$ \\
\hline $\begin{array}{l}\text { Flight towers } \\
\text { (TKR ID B and 1-15) }\end{array}$ & $\begin{array}{l}6.8 g(Z) \\
4.4 g(X, Y)\end{array}$ & $\begin{array}{l}6.1 g \operatorname{RMS}(Z) \\
6.6 g \operatorname{RMS}(X, Y)\end{array}$ & $\begin{array}{l}4 \text { cycles } \\
-15 \text { to }+45^{\circ} \mathrm{C} \\
\text { Max. rate }= \pm 20^{\circ} \mathrm{C} / \mathrm{h}\end{array}$ \\
\hline
\end{tabular}

Dynamic test levels were performed separately both along the thrust $(Z)$ and lateral $(X, Y)$ directions. The sine levels are the maximum loads applied in the test frequency range.

\subsection{Dynamic tests}

The dynamic tests on panels were performed on the trays of the engineering model tower and of the proto-flight tower. The panels were subjected to dynamic solicitations only along the thrust direction, since no modes were expected along the lateral directions in the frequency range required [18]. The test sequence includes a random vibration test preceded and followed by a sine survey.

The goal of the test on panels is twofold:

(1) to study the normal modes and verify that the panel resonance frequencies are greater than the minimum required values;

(2) to verify the workmanship of these components by exposing the items to a random vibration environment and by checking that the post-random low level signature response remains in the required margins.

The tests on panels were performed at Centrotecnica, in Milan, a company specialized in dynamic test activity using an electrodynamic shaker (slip table) LDS V864 LT. The slip table was equipped with an expander $600 \times 600 \times$ $270 \mathrm{~mm}^{3}$ to allow the vibration test along the axis of interest. The attachment of the panel to the expander was provided by a fixture consisting of four L-shaped aluminum pieces that simulated the attachment to the sidewalls. The L-shaped pieces were screwed on an aluminum square plate that provided the interface with the shaker expander.

The response of the structure was measured by a 3-axial accelerometer located in the center of the bottom surface (tungsten side) of the panel, mounted upsidedown on the shaker table. Additional accelerometers located in the corner of the L-shaped pieces were used to control the shaker input. Fig. 4 shows the test set-up, including the top of the shaker and expander, the

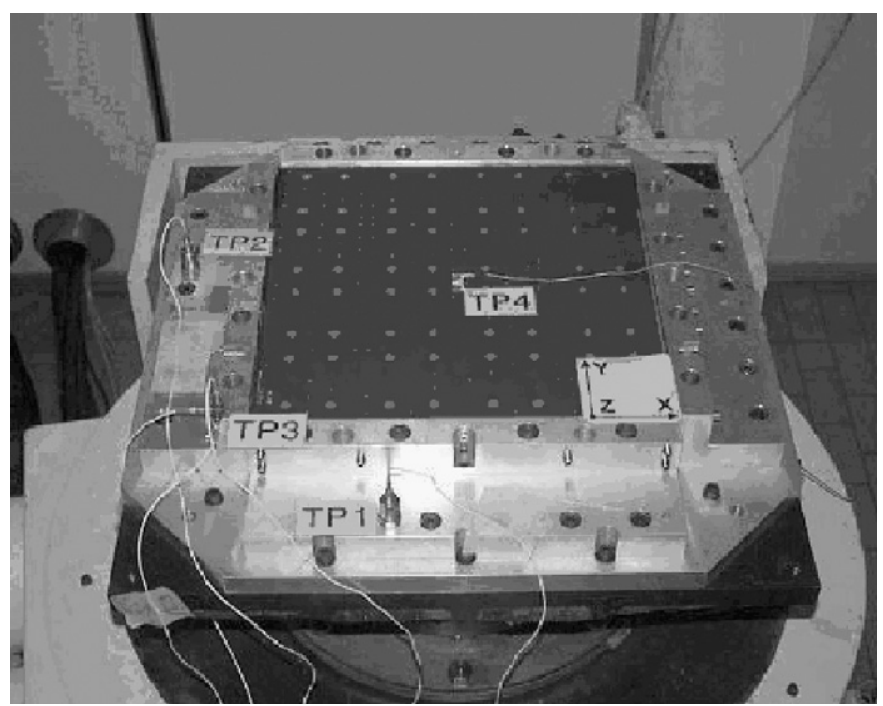

Fig. 4. Vibration test set-up for panels. The visible surface of the panel is the kapton bias circuit. The control accelerometers are labelled TP1, TP2 and TP3, while the measurement accelerometer is TP4.

aluminum square plate and fixture, and the panel equipped with accelerometers.

The resonance search before and after random vibration is intended to discover possible structural degradation induced by the random vibration test. In case of degradation, the overall stiffness is reduced and consequently the resonance frequencies decrease. In the resonance search, an input target amplitude of $0.25 g\left(g \simeq 9.81 \mathrm{~m} / \mathrm{s}^{2}\right.$ is the standard gravity acceleration) was selected as the minimum acceleration level to identify the transfer function and measure the response up to $2000 \mathrm{~Hz}$. The random vibration excited the panel structure to $14 \mathrm{~g}$ RMS for the engineering model panels and $10 \mathrm{~g}$ RMS for the proto-flight ones. All the tests performed have shown excellent results 
and normal modes higher than the minimum required frequency for the first mode (i.e. $>600 \mathrm{~Hz}$ for a mid tray with $2.7 \%$ r.l. tungsten converter).

Fig. 5 shows an example of the result of a low level signature sweep performed after the random test, measured by the accelerometer located in the center of the panel along the vertical axis (thrust). In this case, the panel was subjected to an ASD spectrum with an overall of $10 g$ RMS random vibration input, and consequently the output spectrum reached an overall level of about $40 \mathrm{~g}$ RMS. Table 4 reports the summary values of the first mode for the five different panel types. The maximum tolerable frequency shift for the first mode after and before the random test was required to be less than $3 \%$ to consider a test successful. All the tested panels were within the required limits. Some of them have shown a frequency shift not greater than $1 \%$. Moreover, after the tests none of the panels showed any kind of degradation, and no residual carbon dust was ever observed.

An ESPI (Electronic Speckle Pattern Interferometry) screening test was also performed on the proto-flight and

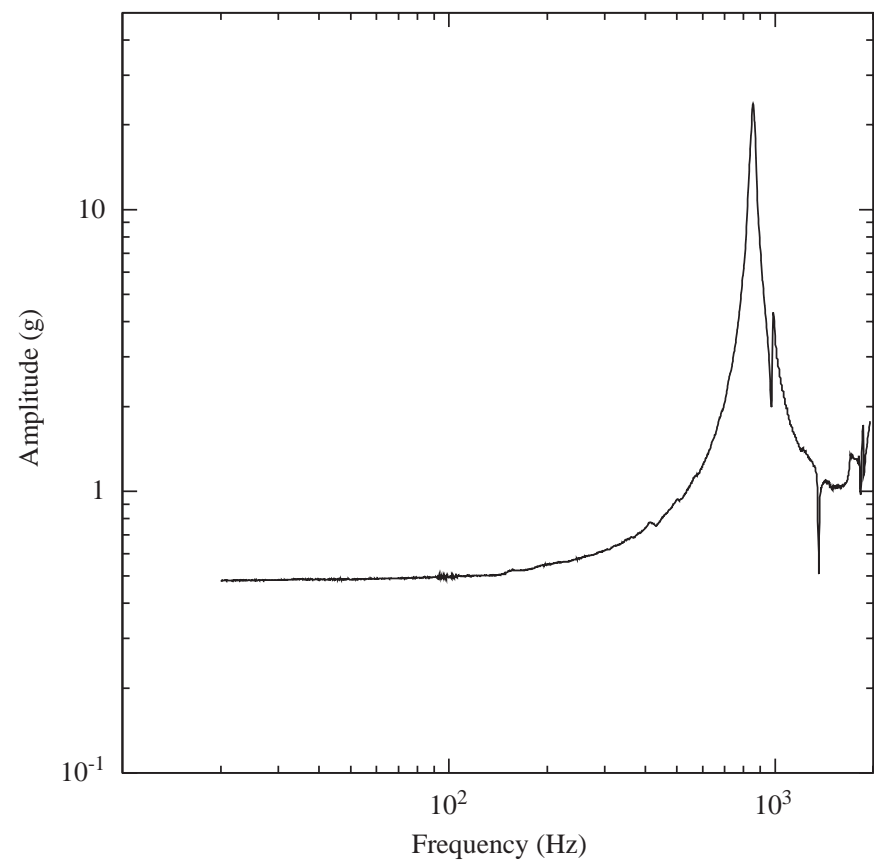

Fig. 5. Low level signature sweep results of a mid panel: response of the accelerometer located in the center of the panel along the vertical direction.

Table 4

Summary of the average values of first mode frequency and corresponding $Q$ measured for the five different panel types

\begin{tabular}{lrr}
\hline Tray panel type & First mode $(\mathrm{Hz})$ & $Q$ \\
\hline Top & 790 & 42 \\
Mid with thin $W$ foil & 900 & 45 \\
Mid with thick $W$ foil & 700 & 78 \\
Mid with no $W$ foil & 1170 & 19 \\
Bottom & 1470 & 12 \\
\hline
\end{tabular}

flight panels by exposing them to a moderate heat source and to a modulated sound woofer [19]. Both dynamic and ESPI tests have demonstrated very reliable detector workmanship and have provided a high level of confidence in the tray assembly procedure. These successful results, which verified both the workmanship and the normal modes for panels, led to the decision of skipping the vibration tests at this level of assembly during the flight production phase, while the ESPI performance continued during the whole flight production phase.

\subsection{Static tests}

A static test was also separately performed on the bottom panels. Static loads up to $8 \mathrm{kN}$ were applied to verify the workmanship of the fabrication processes and to demonstrate the static strength and stability of the structural assembly, such as the bonding of the flexures and corner brackets onto the panels [20]. All the bottom panels passed the static tests without any issue.

\subsection{Thermal tests}

Thermal cycling between two temperature extremes (see Table 2) is performed to verify the workmanship of hardware items that may be susceptible to thermally induced mechanical fatigue. ${ }^{1}$ Thermal cycles were performed at room pressure in a nitrogen environment on all trays already equipped with silicon sensors and electronics [22].

The goal of the thermal test on functional trays was to verify the workmanship of the trays and to demonstrate that the silicon sensors withstand the thermal gradients without degradation of functionality prior to their assembly into the tower. An electrical-functional test performed before and after the thermal cycling was needed to show that the functional capability of the hardware was not diminished. Thermal cycles on engineering model trays were performed according to the complete sequence, but without any functional test. Visual inspections were used to verify that no mechanical damage had occurred.

The thermal tests on trays were performed in INFN labs, where large volume climatic chambers [23] were used to accommodate up to 12 trays at a time. Thermal tests were performed on engineering model, proto-flight and flight trays after the assembly of silicon detectors. Each tray was housed in an aluminum service box [24], provided with a plug for nitrogen flow, connector savers, an ESD connection point, and a protecting gasket to prevent dust from entering the box. The boxes were used to ensure safe transportation and testing, while maintaining the required cleanliness and relative humidity levels.

\footnotetext{
${ }^{1}$ The panels were also exposed to vacuum $\left(P<10^{2} \mathrm{~Pa}\right)$ at $60^{\circ} \mathrm{C}$ for $24 \mathrm{~h}$ to check the kapton bias circuit adhesion and to identify eventual delamination under vacuum due to air trapping by visual inspection [21].
} 
The temperature experienced by the flight trays ranged in four cycles from -15 to $+45^{\circ} \mathrm{C}$, with a rate of change of $\pm 40^{\circ} \mathrm{C} / \mathrm{h}$ and a soak period of $2.5 \mathrm{~h}$ at each extreme of one cycle. The trays belonging to the proto-flight tower were exposed to thermal cycles from -20 to $+55^{\circ} \mathrm{C}$, while the trays of the engineering model tower were cycled between -30 and $+50^{\circ} \mathrm{C}$. The temperature ranges applied to all the trays are summarized in Table 2.

The tests were carried out at room pressure in a nitrogen environment. During the test, nitrogen gas was flowed into the boxes containing the trays at very low rate, just enough to maintain a $\mathrm{N}_{2}$ environment but not provide cooling or heating of the tray. In addition, before entering the boxes, the nitrogen gas from the tank is flowed through a steel cylinder located in the chamber in order to exchange heat and bring the nitrogen to the temperature of the chamber before entering the boxes. All the boxes inside the climatic chamber were connected to the cylinder with independent tubes. Each box was equipped with a thermocouple attached to the top surface in order to monitor the temperature outside the box. All the trays tested successfully underwent the thermal cycles.

\subsection{Stacked cosmic rays tests and tower assembly}

Functional tests at tray level were performed in conjunction with the thermal cycles by stacking the trays in a rack [25]. The trays, always enclosed in the service boxes and stacked on the rack, were connected to a TEMbased data acquisition system and tested by reconstructing and monitoring cosmic-ray tracks passing through the stack. All flight trays were also tested to verify that each of them draws the correct quiescent power and responds correctly to all command sequences from the TEM. The TKR assembly followed the stacked cosmic rays test.

\section{TKR environmental tests}

The environmental tests on TKRs were carried out in an external company. Once the TKRs have been assembled in INFN labs and checked for functional verification, they were shipped to the Alenia-Alcatel center in Rome to be subjected to the environmental tests. These tests included dynamic tests and thermal-vacuum cycles (see Table 3). Before, after and during these tests, functional comprehensive and limited performance tests were performed to verify the functionality of the TKR and, when necessary, to identify failures in the electronics chain (as discussed in Section 7).

The engineering model tower was subjected to a unique test sequence, since it was not a full functional tower and no functional tests could be performed. The dynamic test sequence included all the steps addressed in the qualification program. However, a thermal balance was added to the thermal-vacuum test, within one thermalvacuum cycle.

\subsection{Dynamic test: set-up, procedure and test levels}

Two electrodynamic shakers were used to perform the vibration test in the vertical (LDS V984) and horizontal (LDS V994) configuration respectively. Each TKR was mounted on a test fixture [27], simulating the attachment to the Grid as in the flight configuration.

The response to the vibration tests was registered by accelerometers glued on the TKR sidewalls. The vibration test on the engineering model tower was performed using 16 accelerometer channels, while for the proto-flight and flight modules only six channels were used. Fig. 6 shows a drawing of the accelerometers location used for the flight TKRs. The fixture was also instrumented with four threeaxial control accelerometers to measure the input excitation along the test direction. The control strategy was based on the maximum feedback response from these accelerometers. The control signals were also used to abort the proof when necessary: an alarm level of $1 \mathrm{~dB}$ with respect to the input level was set as a warning. The occurrence of a control signal exceeding $4 \mathrm{~dB}$ (abort) with respect to the input level resulted in an immediate halt of the test.

Dynamic tests were performed separately both along the thrust $(Z)$ and lateral $(X, Y)$ directions (axis) [28]. For each axis, the dynamic proofs began with a low level signature sweep, used to evaluate the transfer function of the tower. It was mainly intended to measure the resonant frequencies and the $Q$ factor of each mode. This modal survey was performed in conjunction with sinusoidal and random vibration sequence, before and after each proof, in order to monitor the tower behavior when subjected to the dynamic environment. Changes in mode frequency and/or amplitude between sine sweep tests were used to identify possible structural damage that may have occurred during the

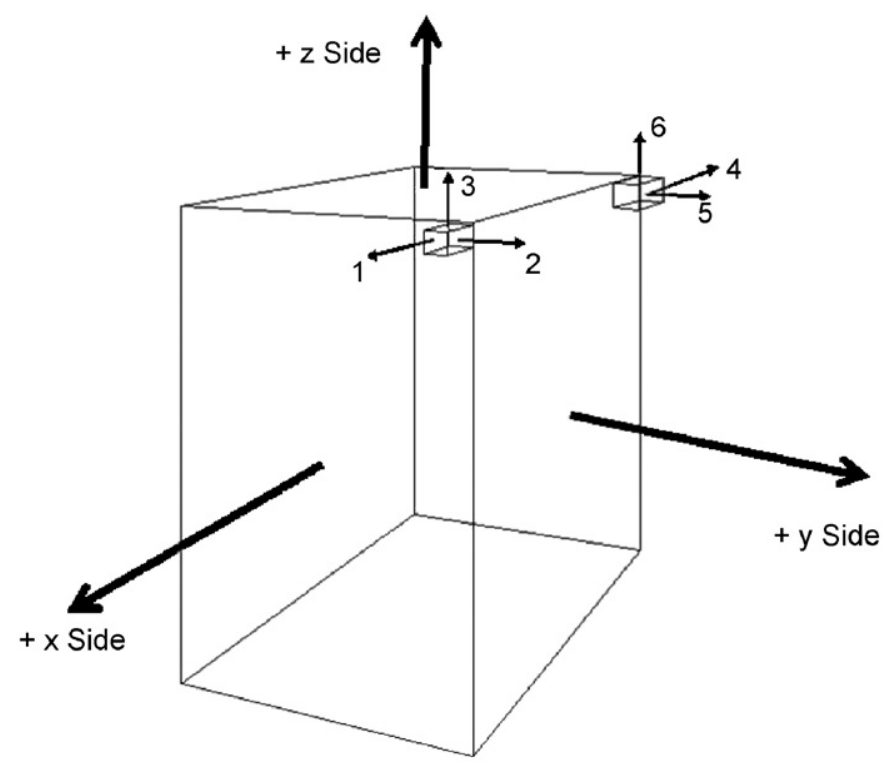

Fig. 6. Schematic drawing of the accelerometers location used for the flight TKRs. The measurement axis are also shown. 
dynamic test sequence. Most important, a comparison of the functional performance between the pre- and postvibration tests completed the dynamic part of the environmental test sequence.

\subsubsection{Sine survey}

The low level signature sweep studies the tower normal modes along the test axis and compares the pre- and posttest results. This proof consisted of a sweep with a fixed input acceleration level from 5 to $2000 \mathrm{~Hz}$ and a sweep rate of 4 octaves per minute (oct $/ \mathrm{min}$ ). The input target amplitude was set to $0.15 \mathrm{~g}$, selected as the minimum acceleration level needed to identify the transfer function and measure the response.

Fig. 7 reports the results of a pre- and post-sequence low level signature sweep along the vertical axis. A summary plot of the first mode frequencies of each tower, along the $Z$ - and $Y$-axis before and after the execution of a complete vibration test sequence, is shown in Fig. 8. The first mode frequency measured on all the TKRs is above $350(130) \mathrm{Hz}$ for the thrust (lateral) axis. These results mean that there is no coupling between the TKR and the LAT structures, since the first mode frequency expected for the LAT is well below $100 \mathrm{~Hz}$ along the $Z$-axis, and the lateral frequency is about $35 \mathrm{~Hz}$. The towers passed the vibration test if the first mode frequency shift was less than $2 \%$ during the dynamic test sequence. A shift greater than $2 \%$ could imply an eventual damage of the structure. In such a case, a more detailed investigation was planned.

The $Q$ values, defined as the ratio between the lowest mode frequency and the corresponding full width at half

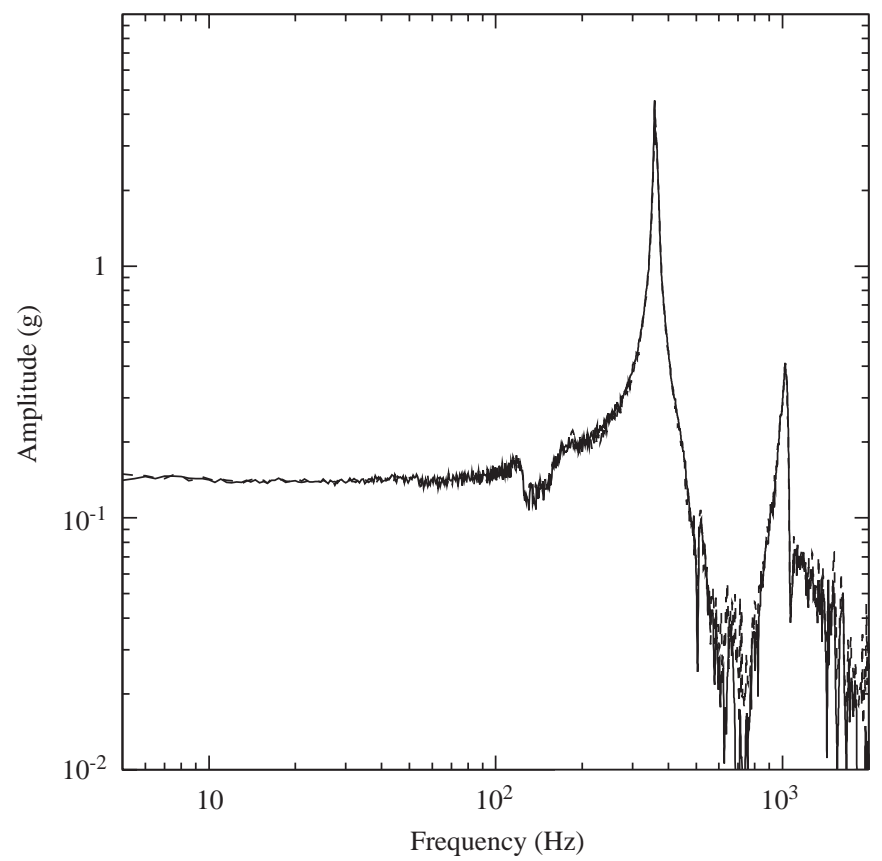

Fig. 7. $Z$-axis low level signature sweep for TKR ID 6. Accelerometer \#3 responses before (dashed line) and after (solid line) the full level random vibration test are superimposed. In this case no frequency shift was observed.

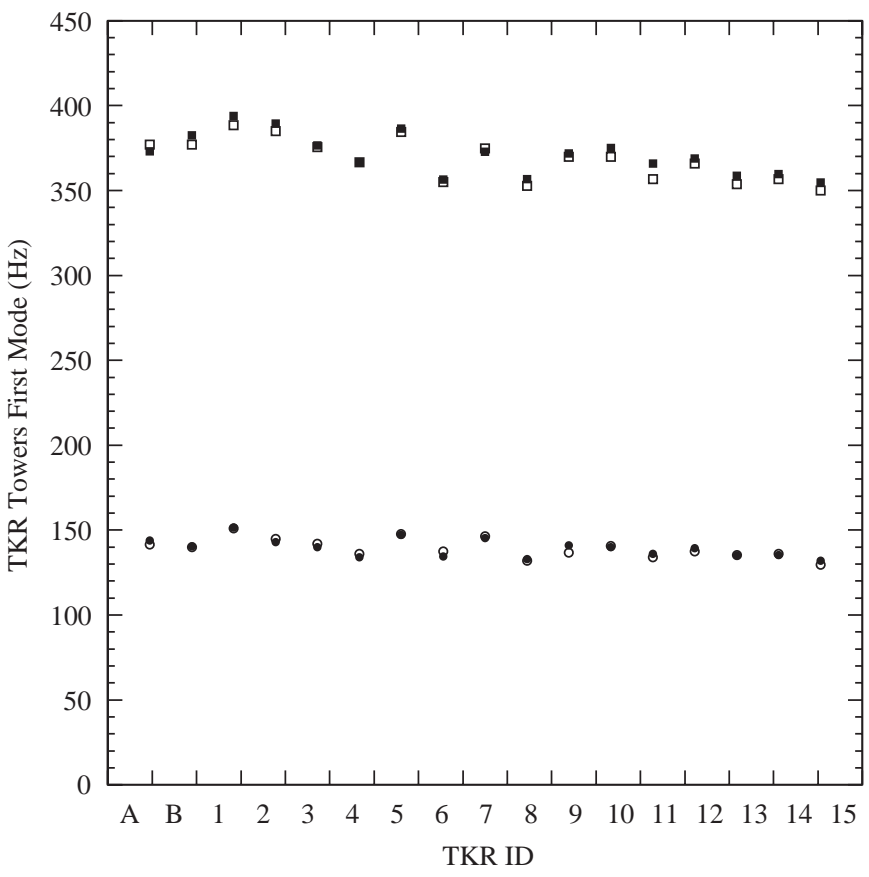

Fig. 8. Low level signature sweep: summary of the first mode frequencies of each TKR. Square symbols: first mode frequency along the $Z$-axis before ( $\mathbf{\square})$ and after $(\square)$ random vibration. Circle symbols: first mode frequency along the $Y$-axis (the $X$-axis results are not shown for they present similar results) before $(\bullet)$ and after $(\circ)$ the random vibration.

maximum, measured on the TKRs were higher than 15 and reached a maximum value of 40 . The damping of the structure is related to the inverse of the $Q$, then the damping measured on the TKRs ranges from about $1 \%$ to about $3 \%$. If a large damping was observed an additional investigation was planned too.

\subsubsection{Sinusoidal vibration and quasi-static test}

The sinusoidal vibration tests the ability of the item to survive the low frequency launch environment, including the static vertical acceleration of the rocket and is intended to provide a workmanship test for the hardware exposed to such loads. Sine vibrations are induced in the payload by the natural frequencies of the bearing structures (satellite, space platforms and launchers). Those frequencies are usually rather low (less than $50 \mathrm{~Hz}$ ). Since the normal modes of the TKR are expected to be higher than $130 \mathrm{~Hz}$ along the lateral axis and $350 \mathrm{~Hz}$ along the thrust direction, no significant response was expected from this test.

During the test of the engineering model a thorough verification was done performing two separate runs [29]. The first (Run 1) was a sine test between 5 and $50 \mathrm{~Hz}$ with a sweep rate of 2 oct $/ \mathrm{min}$, according to the spectrum profile values reported in Table 5. The second was a quasi-static acceleration test in a narrower range (from 25 to $35 \mathrm{~Hz}$ ) at a constant load of $5.9 \mathrm{~g}$ along the vertical direction and $1.9 \mathrm{~g}$ along the horizontal axis, with a slower sweep rate $(0.75 \mathrm{oct} / \mathrm{min})$. In particular, the loads applied in the second run simulated the shocks experienced by the payload in the main engine cut-off phase during the launch. 
Table 5

Engineering model: sinusoidal vibration test levels

\begin{tabular}{|c|c|c|c|}
\hline Axis & $\begin{array}{l}\text { Frequency } \\
(\mathrm{Hz})\end{array}$ & $\begin{array}{l}\text { Acceleration } \\
\text { level }(\mathrm{g})\end{array}$ & $\begin{array}{l}\text { Sweep rate } \\
(\text { Oct } / \mathrm{min})\end{array}$ \\
\hline \multirow[t]{4}{*}{ Thrust (Z) Run 1} & $5-5.5$ & $\begin{array}{l}40 \mathrm{~mm} \text { double } \\
\text { amplitude } \\
\text { displacement }\end{array}$ & 2.00 \\
\hline & $5.5-20$ & 2.40 & 2.00 \\
\hline & $25-35$ & 8.5 & 2.00 \\
\hline & $40-50$ & 2.00 & 2.00 \\
\hline Thrust (Run 2) & $25-35$ & 5.90 & 0.75 \\
\hline \multirow{7}{*}{$\begin{array}{l}\text { Lateral }(Y-X) \\
\text { Run } 1\end{array}$} & $5-8.3$ & $40 \mathrm{~mm}$ double & 2.00 \\
\hline & & $\begin{array}{l}\text { amplitude } \\
\text { displacement }\end{array}$ & \\
\hline & $8.3-15$ & 5.5 & 2.00 \\
\hline & $15-25$ & 1.40 & 2.00 \\
\hline & $25-35$ & 0.10 & 2.00 \\
\hline & $35-40$ & 1.40 & 2.00 \\
\hline & $40-50$ & 2.30 & 2.00 \\
\hline Lateral (Run 2) & $25-35$ & 1.40 & 0.75 \\
\hline
\end{tabular}

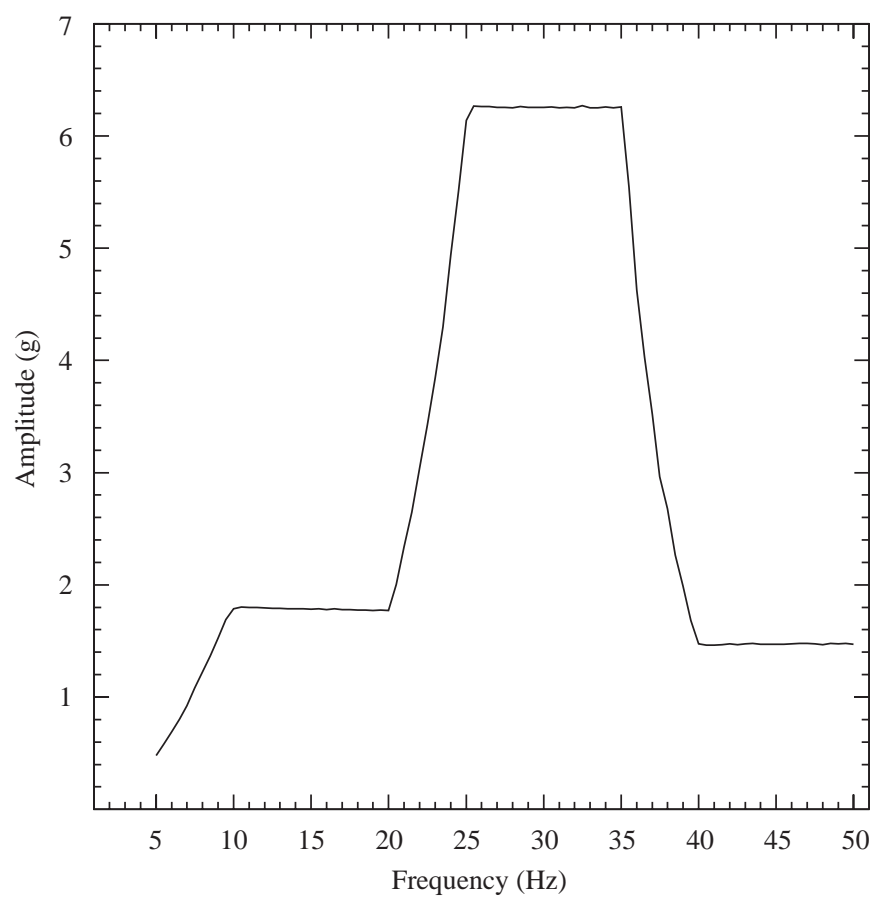

Fig. 9. TKR $Z$-axis test sine profile output measured by the accelerometer \#3.

For the proto-flight tower, the spectra used in the two runs above were merged using, for each frequency range, the highest load level among the two, in order to perform just one run with a sweep rate of $4 \mathrm{oct} / \mathrm{min}$.

Each flight tower was also exposed to a sinusoidal vibration test at acceptance levels in the frequency range from 5 to $50 \mathrm{~Hz}$ in both thrust and lateral directions. As for the proto-flight tower, the quasi-static test was included in the sinusoidal run, with change in the sweep rate of $4 \mathrm{oct} / \mathrm{min}$. Fig. 9 shows the output spectrum measured by the accelerometer \#3 (Fig. 6) during the $Z$-axis test case.
Since no differences were observed between input loads and output response, then the profile shown in this figure represents the input spectrum. The maximum quasi-static loads were $6.8 \mathrm{~g}(25-35 \mathrm{~Hz})$ along the $Z$-axis and $4.4 \mathrm{~g}$ $(5-15 \mathrm{~Hz})$ along the horizontal axis. As expected, in no case did the TKRs show a resonant response at low frequency and no change in the lowest mode was observed between the pre- and post-test modal survey. These results confirmed that all the modules are able to survive the mechanical stresses expected during the launch.

\subsubsection{Random vibration}

The random vibration is the main dynamic test, designed to study the structural response when an item is subjected to the launch environment. The random vibration test is performed by using an ASD spectrum that includes the acoustic inputs plus the effects of vibration transmitted from the rocket (Delta II Heavy in the GLAST case) through the structure during the lift-off.

Random vibration tests on all the towers have been done along all three axes with input levels of $6.1 \mathrm{~g}$ RMS for the vertical axis and $6.7 \mathrm{~g}$ RMS for the lateral directions, with a notching of the original random spectrum around the resonant frequencies. This kind of force-limiting strategy is foreseen in the test plans and procedures since the input random level expected for the TKRs is below the GEVS workmanship level. As a consequence, the notching procedure is recommended to avoid over-testing of the structure in the frequency bands including the first mode of the tower

The notches applied are based on the analytical fundamental mode predictions in the loaded directions. The notch center frequency has been adjusted to the measured fundamental frequencies from the results of the low level runs. Moreover the width of the notch has been set according to the experimental results as well as to the maximum slope of the shaker controller.

For the tests of the engineering model and the protoflight towers, four successive runs were performed at different increasing levels: the first at $-12 \mathrm{~dB}$ with respect to the maximum level, the second at $-6 \mathrm{~dB}$, and the third at $-3 \mathrm{~dB}$, with a duration of $30 \mathrm{~s}$ each. The fourth run at the workmanship level was performed for $120 \mathrm{~s}$ for the engineering model and $60 \mathrm{~s}$ for the proto-flight tower. Each run was preceded and followed by a low level signature sweep (post test signature) to check for any change in resonance frequency due to the application of the random spectrum.

The first test on an engineering model tower was performed in December 2003. The main goal of validating the towers design was not achieved, since the random vibration test failed due to shifts in the fundamental frequencies and due to response amplitudes greater than the allowed values (Section 6.1.1). A hardware problem was identified in the attachment of the flexures to the grid interface. Alignment tolerances required the bolt holes to be substantially larger than the bolts, such that the joints 
had to be held stationary by friction. The random vibration caused movement and resulted in several of the bolts losing torque and backing out. Consequently, the design of the interface was reviewed and modified to use pairs of eccentric cone bushing to allow tight, zero-tolerance joints to be made while maintaining accurate alignment between the Grid and the Tracker [8]. The engineering model tower in the new design was re-tested in June 2004 and successfully passed.

The random vibration tests sequence at acceptance level for the flight towers called for the performance of one random vibration run starting from lower levels $(-12 \mathrm{~dB}$ and $-6 \mathrm{~dB}$ ) for $30 \mathrm{~s}$ before applying the full level random vibration test for $1 \mathrm{~min}$. An example of a random vibration test response measured by the accelerometer \#3 at full level along the $Z$-axis is shown in Fig. 10. The input spectrum is superimposed in the same figure. The input spectrum applied during the lateral axis ( $X$ and $Y$ ) tests is similar to the $Z$-axis one, but the notching is centered around the expected lateral first mode frequency $(130 \mathrm{~Hz})$.

\subsection{Thermal-vacuum}

The thermal design must essentially consider an energy balance of a system, whether of a component or a complete instrument. This includes external heating from the Sun, Earth and other planets, combined with internal heat generation by the instrument. Realistic thermal environments are assessed to determine which worst-case conditions will stress the hardware the most. Factors include time of year, sun-orbit orientation, eclipse duration, operational mode, time of mission (beginning or endof-life), and surface degradation. Worst-case conditions are

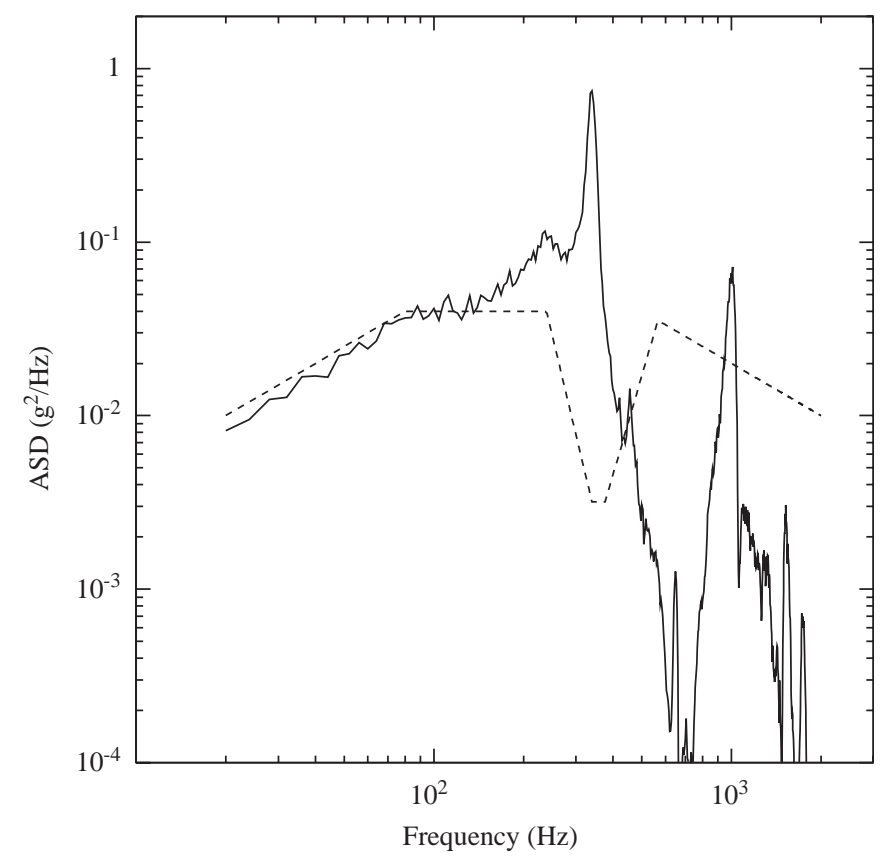

Fig. 10. $Z$-axis random vibration test on the TKR ID 6. Dashed line: notched input ASD spectrum. Solid line: response of the accelerometer \#3. used in thermal analytic models to predict the hot and cold temperature extremes that the instrument may experience during its mission life. These temperature extremes establish the basis for the test environments [30]. There are two different types of thermal-vacuum tests: a thermal balance test and thermal-vacuum cycles.

As already discussed in Section 3, the Tracker in the LAT configuration transfers its waste heat mainly through the thermal coupling to the Grid, then through heat pipes that transport heat off the Grid [31], to be ultimately radiated to space. The TKRs were designed to conduct heat through the sidewalls to the bottom tray. A thermal balance test was carried out on the engineering model and proto-flight tower to verify the thermal design. Thermalvacuum cycles on the flight hardware were performed to demonstrate that the TKRs will survive the thermal gradients expected during the mission and that the functional capability of the hardware is not degraded by thermal transients. A thermal-vacuum test subjects a test article to a number of thermal cycles in a vacuum environment. This test is the most realistic ground simulation of a flight environment, so performance verification is a primary objective, accomplished through functional tests at hot and cold temperature plateaus.

Each thermal-vacuum test on a TKR consisted of four thermal cycles between -15 and $+45^{\circ} \mathrm{C}$ at a vacuum level of $10^{-3} \mathrm{~Pa}$ (about $10^{-5}$ Torr). Functional tests were carried out during transitions and at hot/cold plateaus to check the silicon strip detectors performance as a function of temperature. Moreover, at each plateau the turn-off/ turn-on capability of the instrument was also verified. The temperature of the TKRs was measured by means of thermocouples located on the external sidewalls, on the top and bottom trays as well as by means of the 16 thermistors located on the eight tower cables. In addition, the protoflight tower was subjected to a thermal balance test. The engineering model tower was subjected to a unique thermal-vacuum cycle between -15 and $+25^{\circ} \mathrm{C}$, since the main goal of that test was the performance of a thermal balance.

\subsubsection{Thermal balance}

A thermal balance test is performed to verify the adequacy of the thermal design. This test allows to verify the temperature distribution in the TKR as predicted by the thermal math model, and in particular, the thermal gradient between the top and the bottom trays, once the thermal equilibrium is reached in a specified environmental set-up. The TKR was defined to be in thermal equilibrium when the rate of temperature change was less than $0.1-0.2^{\circ} \mathrm{C} / \mathrm{h}$ for a period of at least $4 \mathrm{~h}$. This temperature change rate represents an energy balance accounting for 90-95\% of the system's heat flow. A single TKR thermal balance test was particularly challenging since the TKR's uncontrolled radiation loss was the same order of magnitude as the tower's waste heat generation. For the engineering model tower, the thermal balance test was 
performed by using 100 thermocouples throughout the TKR to measure its temperature distribution, for a combination of three Tracker power levels and three controlled base plate temperatures. The complete test lasted two weeks, operating $24 \mathrm{~h}$ per day. The plan, procedure and test results are reported in Ref. [32].

The engineering model thermal balance test was carried out in an Alenia thermal-vacuum chamber consisting of a stainless steel cylinder with an inside diameter of about $4 \mathrm{~m}$ and a length of $4.5 \mathrm{~m}$, reaching a vacuum level up to $10^{-4} \mathrm{~Pa}$. The internal walls (shrouds) of the chamber were filled with liquid nitrogen to provide the cooling of the chamber environment. The chamber was also equipped with a decontamination plate to trap out-gassing particles.

The tower module was assembled in the chamber by using the chamber rails supporting appropriate Mechanical Ground Support Equipment consisting of:

(1) a base plate: a black painted aluminum plate acting as an interface between the tower and the chamber environment in order to drive the temperature of the test item. The base plate was assembled above the rails and was thermally decoupled from it by means of NEMA G10 pieces. The plate was painted black to increase the heat exchange between it and the chamber shrouds. A series of strip/tape heaters ( $1000 \mathrm{~W}$ total) located on the top surface of the base plate provided heat to the plate, thus controlling its temperature;

(2) a grid ring that is the interface between the base plate and the tower. The tower was attached to it, both mechanically, by titanium flexures, and thermally, by copper straps, just as in the flight operation design. The grid ring was bolted to the base plate to assure good heat transfer to that item and to minimize the thermal gradient between them;

(3) a thermal MLI (Multi-Layer Insulation) blanket wrapped around the tower to prevent radiation heat transfer with the chamber environment;

(4) a guard shield, surrounding the MLI blanket to control the heat exchange between the tower and the chamber walls during the thermal balance tests. To achieve this, each surface of the guard shield was uniformly instrumented with heaters used to balance the radiation loss from the blanket. The power dissipated by the heaters was adjusted in order to set the average temperature on the guard shield close to the average temperature on the tower.

A non-flight design TEM installed below the cold base plate provided voltage from the power supply to the tower, by means of the eight flex cables, and the read-out of the thermistors located on the cables, as in the flight design. The temperature measurements were made by a custom acquisition system using National Instruments SCXI modules reading the voltage signals from the thermistors and thermocouples.
One thermal cycle between $-15^{\circ} \mathrm{C}$ and $+25^{\circ} \mathrm{C}$ was performed for the engineering module. The cycle included seven thermal balance (TB) cases performed at different power levels and base plate temperature configurations. Three Tracker power levels 12, 10 (nominal) and $8 \mathrm{~W}$ were combined with different base plate temperatures 20,0 and $-15^{\circ} \mathrm{C}$ to bracket the maximum range expected in flight operations.

Evaluation of the TKR temperature was done along its height, from the bottom to the top, dividing it into six main parts representing the nodes used for the temperature predictions of the Tracker. The first node corresponds to the grid ring, the second to the bottom tray of the tower, defined by the average temperature among all the thermocouples positioned on this tray, while the seventh node is defined by all the thermocouples positioned on the top tray and corresponding sidewalls. Fig. 11 shows the results of the thermal balance test: the average temperature of each node for each thermal balance case, starting from the grid ring and going up to the top tray. The black dots correspond to the grid ring temperature. In the $12 \mathrm{~W}$, $20^{\circ} \mathrm{C}$ thermal balance case, the temperature gradient between the bottom and the top trays was $6.5^{\circ} \mathrm{C}$, while it was about $6^{\circ} \mathrm{C}$ in the $8 \mathrm{~W}$ case at the same grid ring temperature.

The measured temperature distribution in the tower is in agreement with the thermal model predictions based on finite element analysis [26]. The vertical thermal gradient in the tower is due to the uniformly generated heat in the trays being conducted down the tower sidewalls to the

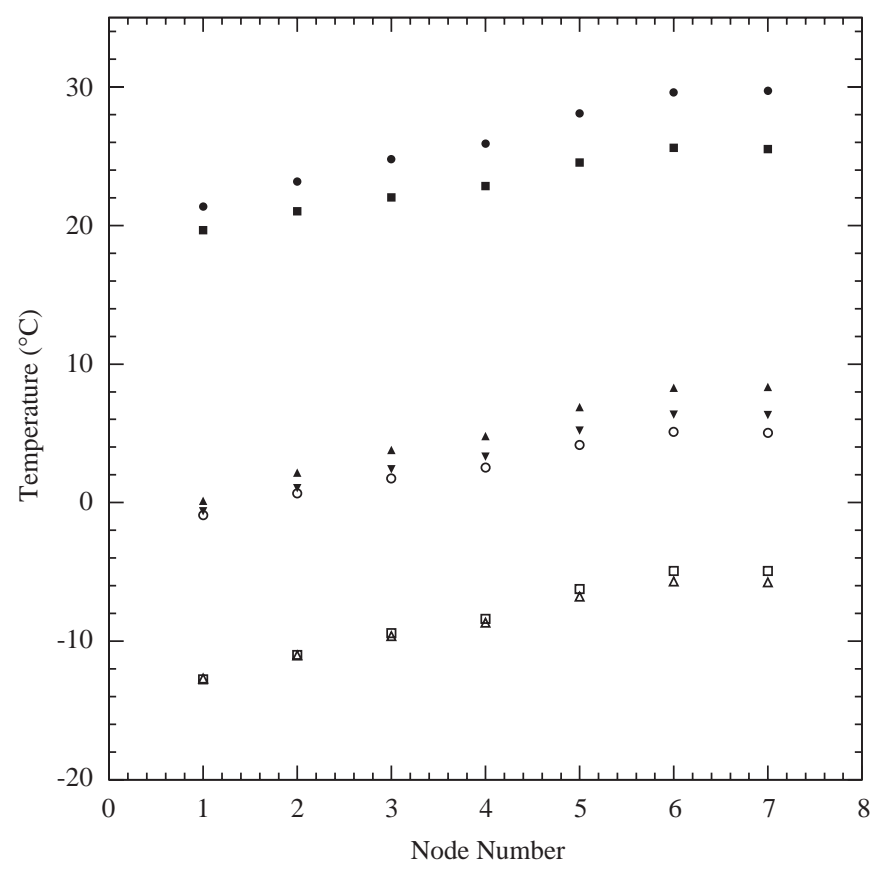

Fig. 11. Average temperature of the nodes for each Thermal Balance case (TB). Node 1 corresponds to the grid ring, node 2 to the bottom tray, node

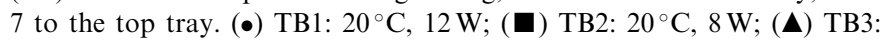
$0{ }^{\circ} \mathrm{C}, 12 \mathrm{~W} ;(\boldsymbol{\nabla}) \mathrm{TB} 4: 0{ }^{\circ} \mathrm{C}, 10 \mathrm{~W}$; (о) $\mathrm{TB} 5: 0{ }^{\circ} \mathrm{C}, 8 \mathrm{~W} ;(\square) \mathrm{TB} 6:-15^{\circ} \mathrm{C}$, $12 \mathrm{~W} ;(\triangle) \mathrm{TB} 7:-15^{\circ} \mathrm{C}, 10 \mathrm{~W}$. 
Grid. The smaller gradient near the top of the tower is due to the finite radiation loss from the top tray, controlled to be the same as predicted when the tower operates on orbit.

A thermal balance test was also performed on the protoflight tower, by using the same set-up and chamber as for the engineering model, but exchanging the TEM for a flight-design one. The results achieved with the proto-flight tower confirmed those obtained with the engineering model.

\subsubsection{Thermal-vacuum cycles}

Thermal-vacuum cycle tests on all flight TKRs were performed in an Alenia thermal-vacuum chamber, a stainless steel cube of sides of about $3 \mathrm{~m}$. Use of this chamber was dictated by coordination of test facility availability and TKR manufacturing schedule. The chamber was equipped with a square cold plate of sides about $1.2 \mathrm{~m}$, located on the floor of the chamber itself. The cooling and heating was achieved by a fluid system thermally controlled by a chiller system located outside the chamber. Only the proto-flight tower was cycled, after the thermal balance test, in the cylindrical chamber as discussed in the previous section.

The cubic chamber used for the flight hardware housed two TKRs simultaneously. Each TKR was mounted on a grid simulator, the same as used in the dynamic test to mount the tower on the shake table, and the grid simulator was placed on a copper stand [33], which was needed to position the TEM below the TKR as in the flight configuration and provide the interface between the TKR and the cold plate. Fig. 12 shows a picture of the test set-up inside the TV chamber. One of the two copper stands is open to show the electronics module located below the TKR. Two cables coming out of the TKR are also clearly visible. The upper part of the TKR is enclosed in an aluminum inner guard shield [34] whose main function is to eliminate radiation exchanges with the chamber walls. Heaters at the top of the guard shield side walls controlled

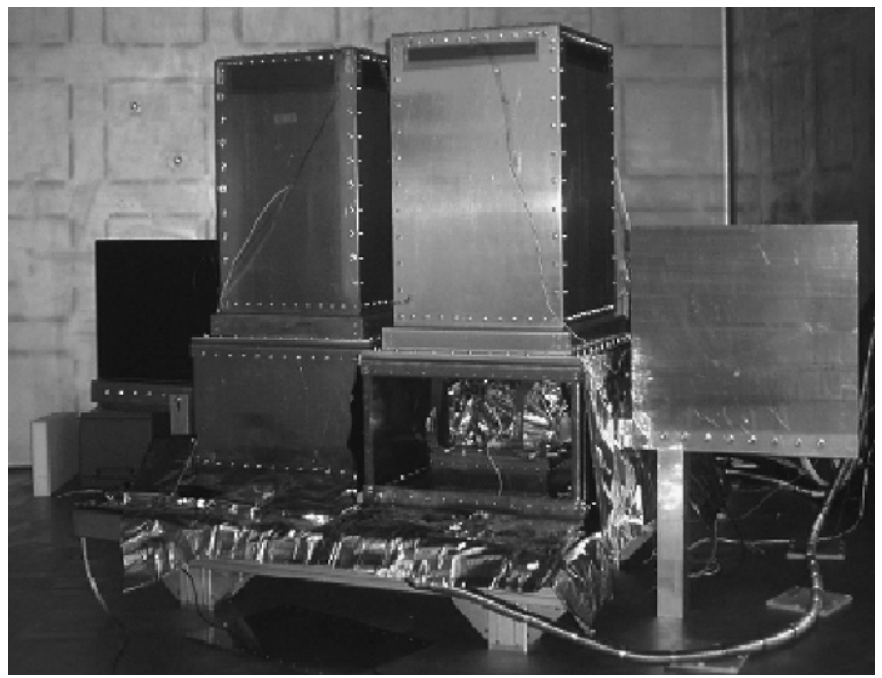

Fig. 12. Thermal-vacuum test set-up. the development of a vertical temperature gradient in the shield walls, matching that of the TKR. Thus, radiation exchange between the TKR and the room temperature chamber walls was eliminated during thermal cycling [35], and the towers operated exactly as on orbit. Also shown in Fig. 12 are the small radiators that thermally connect the electronics modules to the chamber walls, necessary to maintain the modules within their required temperature range.

Once the chamber was closed, the pumping down to the required vacuum level of $10^{-3} \mathrm{~Pa}\left(\sim 10^{-5}\right.$ Torr $)$ was performed while maintaining the test articles at room temperature. When a pressure level close to the final value was reached, the test cycles started and the TKRs were turned on and electrically tested, following the approved procedures. The temperature was measured both by means of the thermocouples placed on the external parts of the TKR (sidewalls, top and bottom trays) and by means of the thermistors located on the cables.

The temperature profile measured by two thermistors located respectively on the bottom and top parts of a tower cable is reported in Fig. 13. The start of the cycles corresponds to the time when the chamber vacuum pressure reached the $10^{-3} \mathrm{~Pa}$ level required for the test and the Tracker power could be turned on for a first functional test (see Section 7). The profile reported shows the whole test with the four cycles between $-15^{\circ} \mathrm{C}$ and $+45^{\circ} \mathrm{C}$ with the hot and cold plateaus at the temperature extremes where functional tests were performed.

The settings to drive the cold plate and, as a consequence, the tower temperature, were set to cycle the

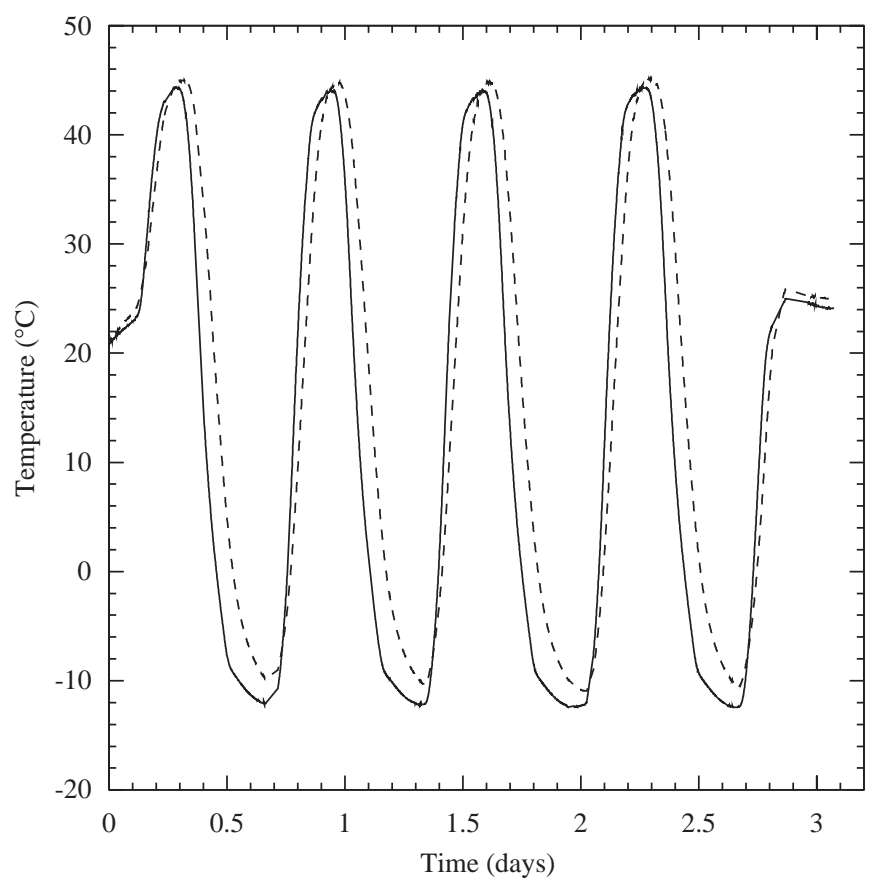

Fig. 13. Temperature profile measured during one thermal vacuum test by two thermistors located respectively on the bottom of the TKR (solid line) and on the top of the TKR (dashed line). 
TKR between the hot and cold temperature extremes with a temperature rate not exceeding $20^{\circ} \mathrm{C} / \mathrm{h}$ in any part of the tower.

\section{Functional tests}

The success of an environmental (dynamic or thermal) test on a TKR requires also the successful performance of functional tests verifying that the tower performance has not degraded during the environmental test sequence. Comprehensive Performance Tests (CPT) and Limited Performance Test (LPT) were planned to verify the functional and performance requirements at the layer and system level [36]. Readout configurations and data and trigger lines were also checked.

The Comprehensive Performance Tests (CPT) include verification of the front-end electronics and registers, monitoring the silicon strip detectors channel by channel and cosmic-ray data acquisition.

The Limited Performance Test (LPT) is a lighter verification of all TKR connections and provides global information on the status of the hardware at the layer level. This test sequence was performed during all of the thermalvacuum cycles, mainly during transitions and at temperature extremes at the end of the $4 \mathrm{~h}$ soak periods.

The CPT provides data on the status of each individual channel as well as system calibrations. The CPT was performed after the vibration test and before, during and after the thermal-vacuum test. In particular, during the thermal-vacuum cycles, the CPT was run at hot and cold plateaus, and the turn-off/turn-on capability of the instrument was also verified.

The environmental tests could cause mechanical damage to the structure, mainly during dynamic excursions, such as de-lamination of the several layers building up the tray (silicon sensors, kapton bias circuit, tungsten layer, carbonfiber face sheet, close-outs) or breaking of the wire bonds between silicon detector channels and electronics or between wafers in a ladder.

The main goal of the functional test was to monitor and locate possible damage in the inside of the TKR that could not be visually inspected. Among the functional tests, the most powerful one is given by the monitoring of the dead and disconnected channels, performed by injecting charge in the calibration chain of each silicon strip channel. Also, the overall TKR efficiency was measured by performing a cosmic-ray test.

The number of not functional channels was continuously monitored during the thermal vacuum cycles. A maximum of 16 bad channels per layer (including dead and disconnected channels) was allowed to consider the test passed.

An additional success criteria is given by the evaluation of the average layer efficiency of the TKR, required to be a minimum of $98 \%$. This is the efficiency to detect a hit from a minimum-ionizing particle passing through the active region of a TKR layer (i.e. excluding the gaps between SSDs and the dead region around the tower edge). A summary of the Tracker performance is reported in Fig. 14: the overall efficiency is above $99.5 \%$ with the exception of the proto-flight tower (TKR ID A), while the bad channel percentage remains below $1 \%$.

\section{Conclusions}

GLAST is a gamma-ray telescope that will be launched in 2008. The Tracker subsystem is one of the main parts of the LAT and has undergone a complex sequence of environmental tests required to verify the capability of the instrument to survive the launch and to survive and operate in on-orbit environments as well as verify the detector performance in the same conditions.

Nineteen TKRs (one engineering model, one protoflight, 15 flight, one spare and one non-flight) were

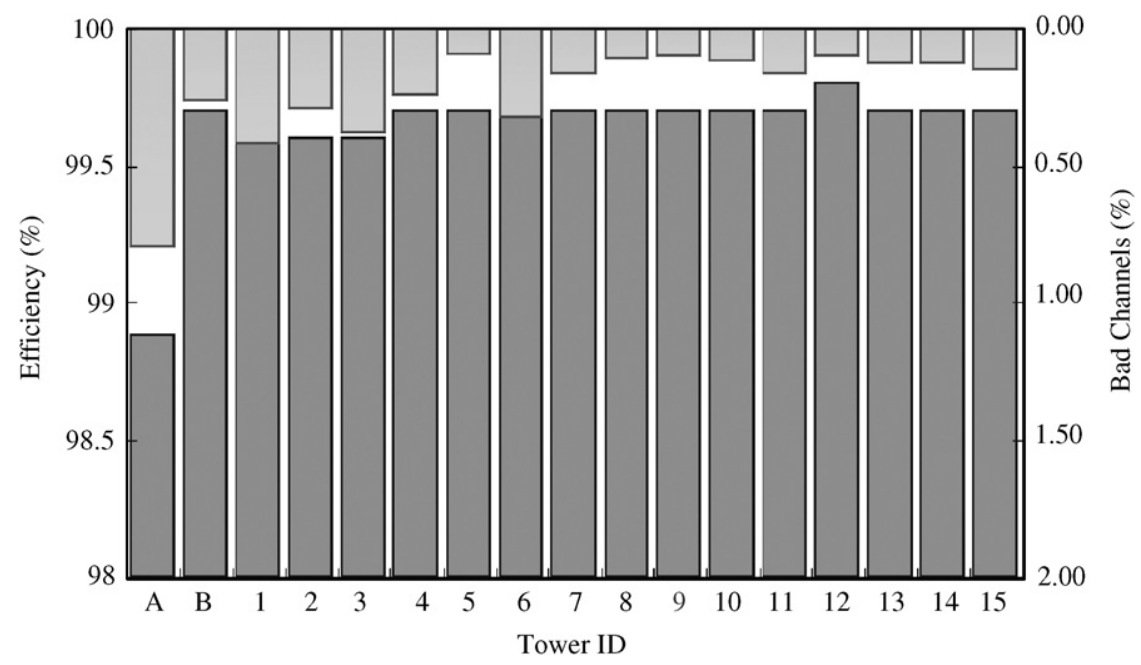

Fig. 14. Summary of the Tracker performance: overall efficiency (dark grey boxes, left $y$-axis) and bad channels percentage (light grey boxes, right $y$-axis) of each tower are reported. 
assembled for the GLAST LAT apparatus. The environmental test campaign started from the engineering model tower in 2003 and was completed in the fall 2005. Eighteen TKRs (all but the non-flight one), and all their trays, successfully passed all the environmental and functional tests maintaining or exceeding the design requirements.

The 16-tower Tracker has been installed in the GLAST LAT science instrument, which has passed all of its environmental and functional tests and is now integrated in the spacecraft.

\section{Acknowledgements}

We would like to acknowledge the engineering teams at SLAC and INFN for their work on the environmental test of the LAT, the LAT integration-and-test team and the electronics group at SLAC for providing the data acquisition hardware and software used to test the Tracker modules, and the LAT integration-and-test team for the assembly of the completed Tracker modules into the LAT and the ensuing functional and environmental testing.

We thank the NASA personnel from the Goddard Space Flight Center who worked to support the Tracker team, E. Grob, T. McCarthy and in particular Tom Venator who worked closely with the team in the U.S. and Italy. We would also like to acknowledge the engineers who supported the environmental test, P. Baird, C. Fransen and B. Pugh. We thank Berrie Giebels for his careful reading of the manuscript and the helpful feedback that he provided.

This work was supported in part by the U.S. Department of Energy, under Contract DE-AC02-76SF00515 and Grants $22428-443410$ and $22428-443410$, by NASA under NAS5-00147, by Istituto Nazionale di Fisica Nucleare (INFN) and by the Agenzia Spaziale Italiana (ASI).

\section{References}

[1] GLAST Science Document, 〈http://glast.gsfc.nasa.gov/science/ resources/docs〉.

[2] W.B. Atwood, Nucl. Instr. and Meth. A 342 (1994) 302.

[3] N. Gehrels, P. Michelson, Astropart. Phys. 11 (1999) 277.

[4] R.C. Hartman, et al., Astrophys. J. Suppl. 123 (1999) 79.

[5] P. Michelson et al., in preparation, Astrophys. J., to be submitted; P. Michelson, The Large Area Telescope, Proceedings of the First GLAST Symposium, AIP Conference Proceedings, vol. 921, 2007, pp. 8-12; GLAST home page: http://www-glast.stanford.edu.

[6] C. Meegan et al., The GLAST Burst Monitor, Proceedings of the First GLAST Symposium, AIP Conference Proceedings, vol. 921, 2007, pp. 13-18; GLAST home page: http://www-glast.stanford.edu.

[7] O. Ferreira, et al., Nucl. Instr. and Meth. A 530 (2004) 323-329.

[8] W.B. Atwood et al., Astropart. Phys. 28 (2007) 422.

[9] A. Brez et al. in preparation, JINST, to be submitted.

[10] M. Nordby, T. Leisgang, LAT environmental specification, LAT-SS00778, GLAST/LAT internal document, unpublished, 〈http://wwwglast.slac.stanford.edu $\rangle$.

[11] GEVS-SE Rev A, June 1996, General environmental verification specification for STS \& ELV, 〈http://arioch.gsfc.nasa.gov/302/ gevs-se/toc.htm $\rangle$.
[12] L. Baldini, et al., Preliminary results of the LAT calibration unit beam tests, Proceedings of the First GLAST Symposium, AIP Conference Proceedings, vol. 921, 2007, pp. 190-204.

[13] R. Bright, LAT program instrument performance verification plan, LAT-MD-00408, GLAST/LAT internal document, unpublished, 〈http://www-glast.slac.stanford.edu $\rangle$.

[14] Centrotecnica s.a.s., Via Meucci 30, Milano, Italy, 〈http://www. ctecnica.it $\rangle$.

[15] R.P. Johnson, et al., LAT tracker MCM functional test and visual inspection procedure, LAT-PS-01971, GLAST/LAT internal document, unpublished, 〈http://www-glast.slac.stanford.edu $\rangle$.

[16] M. Ziegler, R.P. Johnson, P. Amnuaypayoat, Tracker MCM burn-in plan, LAT-TD-02367, GLAST/LAT internal document, unpublished, 〈http://www-glast.slac.stanford.edu .

[17] Alenia-Alcatel, Roma, Italy, Current web page: 〈http://www1. alcatel-lucent.com/space/index.htm $\rangle$.

[18] F. Giordano, M.N. Mazziotta, S. Rainó, TKR Trays-vibration test plan and procedure, LAT-TD-00154, GLAST/LAT internal document, unpublished, 〈http://www-glast.slac.stanford.edu

[19] A. Brez et al., Tracker Tray Test with Electronic Speckle Pattern Interferometry, LAT-PS-1918, GLAST/LAT internal document, unpublished 〈http://www-glast.slac.stanford.edu〉.

[20] N. Saggini, et al., Tracker tray test, bottom tray static test procedure, LAT-PS-03993-04, GLAST/LAT internal document, unpublished, 〈http://www-glast.slac.stanford.edu $\rangle$.

[21] F. Belli, et al., LAT tracker tray panel thermo vacuum test procedure, LAT-PS-03349, GLAST/LAT internal document, unpublished, 〈http://www-glast.slac.stanford.edu〉.

[22] F. Giordano, M.N. Mazziotta, S. Rainó, TKR trays thermal test plan, LAT-TD-01839;

F. Gargano, F. Giordano, M.N. Mazziotta, S. Rainó, TKR trays thermal test procedure, LAT-TD-03651-02, GLAST/LAT internal documents, unpublished, 〈http://www-glast.slac.stanford.edu $\rangle$.

[23] Angelantoni Industrie Spa, Località Cimacolle, Italy, 〈http:// www.angelantoni.it $\rangle$.

[24] M.N. Mazziotta, M. Mongelli, Tray service box, LAT-DS-03738, GLAST/LAT internal document, unpublished, 〈http://www-glast. slac.stanford.edu $\rangle$.

[25] L. Latronico, Stacked trays electrical test plan, LAT-PS-03361, GLAST/LAT internal document, unpublished, $\langle\mathrm{http}: / / \mathrm{www}$-glast. slac.stanford.edu $\rangle$.

[26] J. Goodman, J. Wang, GLAST tracker EM thermal vacuum correlation, LAT-TD-05549, GLAST/LAT internal document, unpublished, 〈http://www-glast.slac.stanford.edu $\rangle$.

[27] B. Bhatnagar, et al., LAT TKR tower assembly shake test mounting plate, LAT-DS-00681-08, GLAST/LAT internal document, unpublished, 〈http://www-glast.slac.stanford.edu .

[28] F. Giordano, M.N. Mazziotta, S. Rainó, et al., Flight TKR towers vibration test plan, LAT-TD-00155;

F. Giordano, M.N. Mazziotta, S. Rainó, Flight TKR towers vibration test procedure, LAT-TD-05499, GLAST/LAT internal document, unpublished, 〈http://www-glast.slac.stanford.edu $\rangle$.

[29] F. Giordano, M.N. Mazziotta, S. Rainó, Engineering module tower vibration test plan, LAT-TD-01841;

F. Giordano, M.N. Mazziotta, S. Rainó, Engineering model tower vibration test procedure, LAT-TD-02363, GLAST/LAT internal documents, unpublished, 〈http://www-glast.slac.stanford.edu $\rangle$.

[30] J. Goodman, et al., Nucl. Instr. and Meth. A 563 (2006) 377.

[31] J. Goodman, M. Nordby, LAT Thermal vacuum test plan, LATMD-01600, GLAST/LAT internal documents, unpublished, 〈http:// www-glast.slac.stanford.edu .

[32] F. Giordano, M.N. Mazziotta, S. Rainó, Engineering module tracker tower thermal balance test plan, LAT-TD-01842;

F. Gargano, F. Giordano, M.N. Mazziotta, S. Rainó, Engineering module tracker tower thermal balance test procedure, LAT-TD02643 ;

F. Gargano, F. Giordano, M.N. Mazziotta, S. Rainó, Report on engineering module tower thermal balance test result, LAT-TD-03609, 
GLAST/LAT internal documents, unpublished, 〈http://www-glast.slac. stanford.edu).

[33] N. Saggini, M.N. Mazziotta, Thermal vacuum MGSE assemblyacceptance test, LAT-DS-05562, GLAST/LAT internal document, unpublished, 〈http://www-glast.slac.stanford.edu .

[34] M. Mongelli, M.N. Mazziotta, Thermal vacuum MGSE assemblyacceptance test-inner guard shield, LAT-DS-05621, GLAST/LAT internal document, unpublished, 〈http://www-glast.slac.stanford.edu $\rangle$.
[35] F. Gargano, F. Giordano, M.N. Mazziotta, S. Rainó, Flight TKR towers thermal vacuum test plan, LAT-TD-01840;

F. Gargano, et al., Flight TKR towers thermal vacuum test procedure, LAT-TD-05500, GLAST/LAT internal documents, unpublished, 〈http://www-glast.slac.stanford.edu $\rangle$.

[36] R.P. Johnson, et al., GLAST LAT TKR tower - electrical test plan, LAT-TD-00191, GLAST/LAT internal document, unpublished, 〈http://www-glast.slac.stanford.edu $\rangle$. 